\title{
Joint medicine-information and pharmacovigilance services could improve detection and communication about drug-safety problems
}

This article was published in the following Dove Press journal:

Drug, Healthcare and Patient Safety

I July 2014

Number of times this article has been viewed

\author{
Jan Schjøtt ${ }^{1-3}$ \\ Jenny Bergman ${ }^{3}$ \\ 'Section of Clinical Pharmacology, \\ Laboratory of Clinical Biochemistry, \\ Haukeland University Hospital, \\ ${ }^{2}$ Institute of Clinical Science, Faculty \\ of Medicine and Dentistry, University \\ of Bergen, ${ }^{3}$ Regional Medicines \\ Information and Pharmacovigilance \\ Centre (RELIS Vest), Haukeland \\ University Hospital, Bergen, Norway
}

Background: RELIS is a Norwegian network of four regional medicine-information and pharmacovigilance centers where pharmacists and clinical pharmacologists provide feedback to health care professionals in spontaneous drug-related questions and adverse drug-reaction (ADR) reports published in a question-answer pair (QAP) database (the RELIS database) and the Norwegian ADR database, respectively.

Objective: To describe the potential of RELIS's dual service to improve detection and communication of drug-safety problems.

Materials and methods: We searched the RELIS database for QAPs about ADRs with use of the Norwegian ADR database as a reference. We also searched the Norwegian ADR database for reports that used the RELIS database as a reference. Both searches were limited to the years 2003-2012. We then selected the example of pregabalin and drug abuse after the marketing of Lyrica in Norway in September 2004 to illustrate RELIS's potential to detect new drug-safety information through a limited number of QAPs and ADR reports.

Results: A total of 5,427 (26\%) of 21,071 QAPs in the RELIS database concerned ADRs. QAPs from this database were used as references in 791 (4\%) of a total of 22,090 reports in the Norwegian ADR database. The Norwegian ADR database was used as a reference in $363(7 \%)$ of 5,427 QAPs that concerned ADRs. Between September 2004 and September 2008, RELIS received eleven questions and 13 ADR reports about suspicion of Lyrica (pregabalin) and different aspects of abuse.

Conclusion: RELIS processes data through two databases that facilitate communication about ADRs. Our service also has the potential to detect new drug-safety problems with a limited number of questions and ADR reports.

Keywords: pharmacovigilance, drug safety, database, pregabalin

\section{Introduction}

Location, organization, and staff competence are of importance for a pharmacovigilance center. ${ }^{1}$ In some countries, the centers are associated with health authorities or drugregulatory agencies. In other countries, national centers cooperate with a network of local centers, part of the health care system, and/or medical universities. ${ }^{2}$ Pharmacovigilance should ideally be closely linked to drug regulation, because governmental support is needed for national and international coordination and communication. ${ }^{1}$ Furthermore, drug-regulatory agencies exchange drug-safety information with pharmaceutical companies, and personnel with regulatory training facilitate both national and international affairs in pharmacovigilance. However, drug safety is not only a regulatory task but represents an important health matter. Local pharmacovigilance centers have a link
Correspondence: Jan Schjøtt

Section of Clinical Pharmacology, Laboratory of Clinical Biochemistry,

Haukeland University Hospital, 5021

Bergen, Norway

Tel +4755973093

Fax +4755 290718

Email jan.didrik.schjott@helse-bergen.no 
to clinical practice and the ability to increase the quality of adverse drug-reaction (ADR) reports. Competence in pharmacy and medicine are often found in local centers that facilitate communication about drug safety with health care professionals. Irrespective of the organization, spontaneous reporting remains a cornerstone of pharmacovigilance, and is of major importance for signal detection. To facilitate the latter, spontaneous ADR reports are processed through national and international databases. ${ }^{3}$

The Norwegian model includes cooperation between the Norwegian Medical Agency and RELIS. RELIS is a network of four regional medicine-information and pharmacovigilance centers. The centers are localized at university hospitals in Norway, where pharmacists and clinical pharmacologists answer spontaneous drug-related questions from health care professionals (mainly physicians and pharmacists) working in hospitals, hospital pharmacies, general practice, and community pharmacies. ${ }^{4,5}$ ADRs are the most common category of questions to RELIS, and represent a substantial part of our question-answer pair (QAP) database (the RELIS database). Use of spontaneous ADR reports from the Norwegian ADR database as references is a possibility in these QAPs. RELIS also handles spontaneous ADR reports from health care professionals, and gives individualized feedback that may refer to QAPs from the RELIS database. ADR reporting is mandatory for physicians and dentists in Norway, and includes reporting on suspicion of fatal and life-threatening ADRs, ADRs that lead to or prolong hospital stay, and ADRs that are new or unexpected. ADR reporting is voluntary for all other health care professionals and consumers. RELIS does signal detection by a qualitative analysis of individual case-safety reports (ICSRs). Signal detection including disproportional analysis is made by the Norwegian Medical Agency. Extensive literature search, pharmacists' and clinical pharmacologists' involvement, and short response time (within 10 calendar days) are important in RELIS's communication about ADRs. Therefore, the mutual use of the aforementioned databases is useful for this communication. In particular cases, detection of new drug-safety information through a joint medicine-information and pharmacovigilance service like RELIS is a possibility, as described here.

\section{Materials and methods}

We searched the RELIS database for QAPs about ADRs with use of the Norwegian ADR database as a reference. We also searched the Norwegian ADR database for reports that used the RELIS database as a reference, using a preexisting module for searches of the reporter-feedback field, which contains the references. Both searches were limited to the years 2003-2012. We then selected the example of pregabalin and suspicion of drug abuse after the marketing of Lyrica ${ }^{\circledR}$ (Pfizer, New York, NY, USA) in Norway in September 2004 to illustrate RELIS's potential to detect new drug-safety information through a limited number of QAPs and ADR reports. All QAPs and ADR reports involving pregabalin from September 2004 until September 2008 were analyzed for any ADR describing aspects of drug abuse or drug dependence without restricting the analysis to selected Medical Dictionary for Regulatory Activities terms. Duplicates between ADR reports and QAPs were excluded based on comparison of the information provided in each report or question.

\section{Results}

Figure 1 shows the results. A total of 5,427 (26\%) of 21,071 QAPs in the RELIS database concerned ADRs. QAPs from this database were used as references in 791 (4\%) of a total of 22,090 reports in the Norwegian ADR database. The Norwegian ADR database was used as a reference in $363(7 \%)$ in a total of 5,427 QAPs that concerned ADRs. Lyrica (pregabalin) was marketed in September 2004 in Norway. In July 2005, RELIS received the first question concerning pregabalin abuse. Two subsequent ADR reports in January 2006 about drug abuse were followed by a question to RELIS in March 2006 suspecting a particular problem among patients with opioid addiction and psychiatric diseases. Descriptions of problems with dose escalation, craving, and withdrawal reactions were found in questions and ADR reports during 2007 and 2008. Eleven questions complemented 13 ADR reports in September 2008 (4 years after marketing) by including a wide spectrum of behaviors indicating drug abuse, eg, the enthusiasm and satisfaction with pregabalin prescriptions shown by former and current drug abusers.

\section{Discussion}

RELIS's dual service with medicine-information and pharmacovigilance could be of importance for two purposes: to meet expectations for feedback in ADR reporting and drug-related queries, and to detect drug-safety problems in particular populations. The mutual use of the two databases as references may at first glance seem limited, but one has to remember that both sources are based on spontaneous and not systematic data collection. In 2003, 47 ADR reports used the RELIS database as a reference compared to 73 in 2012. In 2003, 0 QAPs used 
RELIS database

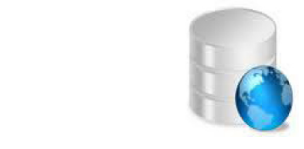

Database of spontaneous question-answer pairs (QAPs)

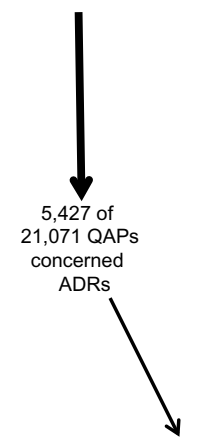

11 QAPs

Drug abuse

Dose escalation Craving Withdrawal reactions Concerns of misuse in patients with opioid addiction and psychiatric diseases Enthusiasm/satisfaction with pregabalin in former or current drug abusers

\section{Norwegian ADR database}

Total results, 2003-2012

Database of spontaneous adverse drug-reaction (ADR) reports $363(7 \%)$ QAPs used the Norwegian ADR database as reference

22,090 ADR reports

Example of pregabalin, 2004-2008

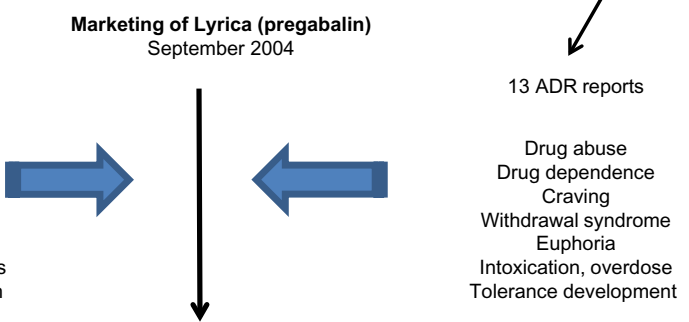

Suspicion of abuse of Lyrica (pregabalin) September 2008

Figure I The Regional Medicines Information and Pharmacovigilance Centres (RELIS) in Norway provide feedback to health care professionals on spontaneous drug-related questions and adverse drug-reaction (ADR) reports published in a question-answer pair (QAP) database (the RELIS database) and the Norwegian ADR database, respectively. Notes: Mutual use of the two ADR information sources has the potential to increase knowledge of drug safety due to data accumulation. The example of pregabalin and suspicion of drug abuse after the marketing of Lyrica in Norway in September 2004 is used to illustrate RELIS's potential to detect new drug-safety information through a limited number of QAPs and ADR reports.

the Norwegian ADR database as a reference compared to 56 in 2012. Therefore, mutual use of the two ADR information sources has the potential to increase due to data accumulation. Importantly, the staff at RELIS process data to both databases, and are thereby alert to suspicion of drug-safety problems, although the number of questions and/or ADR reports is small. The example of pregabalin illustrates that a particular drug-safety issue associated with subgroups of patients could be detected. Four years after marketing in Norway, 13 ADR reports and eleven questions to RELIS depicted a drug-safety problem with pregabalin later described internationally. ${ }^{6-9}$ This is in contrast to the experience that pregabalin is safe, based on results of clinical studies and patient reporting. ${ }^{10,11}$

Pregabalin has also been controversial among physicians in Norway, with discussions of its value in the treatment of neuropathic pain and generalized anxiety weighed against its potential for abuse. ${ }^{12,13}$ Based on staff discussions within the RELIS network and close communication with the Norwegian Medical Agency, matters of concern like the case of pregabalin and abuse were submitted to the Norwegian Pharmacovigilance Advisory Board for further national and international discussions. Furthermore, in 2013 the Norwegian Pharmacovigilance Advisory Board advised the Norwegian Medical Agency to make pregabalin a controlled drug, and its prescription status will be evaluated. ${ }^{14}$

The use of qualitative analysis of individual ICSRs and questions about ADRs on suspicion of drug-safety problems as described here is well known to pharmacovigilance centers and medicine-information centers. Furthermore, databases with ICSRs can be studied with systematic methods. ${ }^{15}$ The present description focused on how the RELIS network with common electronic resources facilitates communication about ADRs, and has the potential to detect new drug-safety problems through an arbitrary method based on a limited number of questions and ADR reports.

\section{Author contributions}

Jenny Bergman performed acquisition and analysis of cases concerning pregabalin. Jan Schjøtt and Jenny Bergman collected data from the RELIS database and the Norwegian ADR database. Jan Schjøtt wrote the manuscript. Both authors contributed to the conception, design, analysis, and 
interpretation of data. Both authors have been involved in drafting the manuscript and revising it critically for important intellectual content, and have given final approval of the version to be published.

\section{Disclosure}

The authors report no conflicts of interest in this work.

\section{References}

1. Uppsala Monitoring Centre. Safety Monitoring of Medicinal Products: Guidelines for Setting up and Running a Pharmacovigilance Centre. Uppsala: Uppsala Monitoring Centre; 2000.

2. Arimone Y, Bidault I, Dutertre JP, et al. Updating the French method for the causality assessment of adverse drug reactions. Therapie. 2013;68(2): 69-76.

3. Oosterhuis I, van Hunsel FP, van Puijenbroek EP. Expectations for feedback in adverse drug reporting by healthcare professionals in the Netherlands. Drug Saf. 2012;35(3):221-232.

4. Schjøtt J, Pomp E, Gedde-Dahl A. Quality and impact of problemoriented drug information: a method to change clinical practice among physicians? Eur J Clin Pharmacol. 2002;57(12):897-902.

5. Schjøtt J1, Reppe LA, Roland PD, Westergren T. A question-answer pair (QAP) database integrated with websites to answer complex questions submitted to the Regional Medicines Information and Pharmacovigilance Centres in Norway (RELIS): a descriptive study. BMJ Open. 2012;2(2):e000642.
6. [No authors listed]. Gabapentin and pregabalin: abuse and addiction. Prescrire Int. 2012;21(128):152-154.

7. Grosshans M, Lemenager T, Vollmert C, et al. Pregabalin abuse among opiate addicted patients. Eur J Clin Pharmacol. 2013;69(12): 2021-2025.

8. Schifano F, D'Offizi S, Piccione M, et al. Is there a recreational misuse potential for pregabalin? Analysis of anecdotal online reports in comparison with related gabapentin and clonazepam data. Psychother Psychosom. 2011;80(2):118-122.

9. Bodén R, Wettermark B, Brandt L, Kieler H. Factors associated with pregabalin dispensing at higher than the approved maximum dose. Eur J Clin Pharmacol. 2014;70(2):197-204.

10. Toth C. Drug safety evaluation of pregabalin. Expert Opin Drug Saf. 2012;11(3):487-502.

11. Härmark L, van Puijenbroek E, Straus S, van Grootheest K. Intensive monitoring of pregabalin: results from an observational, web-based, prospective cohort study in the Netherlands using patients as a source of information. Drug Saf. 2011;34(3):221-231.

12. Westin AA, Bramness JG, Chalabianloo F, Rygnestad T, Slørdal L. [Pregabalin should be moved to the prescription group B]. Tidsskr Nor Laegeforen. 2013;133(6):615-616. Norwegian.

13. Bergsholm P. [Prescription group C correct for pregabalin]. Tidsskr Nor Laegeforen. 2013;133(10):1044-1045. Norwegian.

14. Norwegian Pharmacovigilance Advisory Board. Minutes of meeting of December 11, 2013. Available from: www.legemiddelverket.no/ Bivirkninger/bivirkningsnemnda/referater/Sider/default.aspx. Accessed April 30, 2014.

15. Karimi G, Star K, Norén GN, Hägg S. The impact of duration of treatment on reported time-to-onset in spontaneous reporting systems for pharmacovigilance. PLoS One. 2013;8(7):e68938.

\section{Publish your work in this journal}

Drug, Healthcare and Patient Safety is an international, peer-reviewed open-access journal exploring patient safety issues in the healthcare continuum from diagnostic and screening interventions through to treatment, drug therapy and surgery. The journal is characterized by the rapid reporting of reviews, original research, clinical, epidemiological and

\section{Dovepress}

post-marketing surveillance studies, risk management, health literacy and educational programs across all areas of healthcare delivery. The manuscript management system is completely online and includes a very quick and fair peer-review system. Visit http://www.dovepress.com/ testimonials.php to read real quotes from published authors. 